\title{
Phonological Variation in Hijazi and Cairene Arabic: The Case of the Uvular Stop /q/
}

\author{
Baraa A. Rajab \\ English Language Institute, King Abdulaziz University, Jeddah, Saudi Arabia \\ Email: brajab@kau.edu.sa
}

How to cite this paper: Rajab, B. A. (2021). Phonological Variation in Hijazi and Cairene Arabic: The Case of the Uvular Stop /q/. Open Journal of Modern Linguistics, 11, 883-892.

https://doi.org/10.4236/ojml.2021.116068

Received: October 26, 2021

Accepted: November 28, 2021

Published: December 1, 2021

Copyright (c) 2021 by author(s) and Scientific Research Publishing Inc. This work is licensed under the Creative Commons Attribution International License (CC BY 4.0).

http://creativecommons.org/licenses/by/4.0/

\begin{abstract}
This small scale, qualitatively based, exploratory study aimed at exploring and analysing voice pattern production of eight participants ( 2 male and 6 female) by eliciting their voice patterns generated from reading forty-three words from a word list and also, from reading a short paragraph. Furthermore, the study aimed at exploring the phonological variation in the spoken Arabic of two regions: Jeddah, Saudi Arabia (Hijazi Arabic-HA) and Cairo, Egypt (Cairene Arabic-CA) and also, examines the role of age, sex, educational level and region on this variation. In particular, the study explored the correlation of voice patterns production as they relate to the non-linguistic factors such as age, gender and educational level. The analysis of the gathered qualitative data was carried out by transcribing the recorded data and coding each voice pattern in question. The results indicated that there is more variation in CA speakers than in HA speakers and also, there are some gender influences on phonological production.
\end{abstract}

\section{Keywords}

Phonological Variation, Hijazi Arabic, Cairene Arabic, Diglossia, Modern Standard Arabic

\section{Introduction}

Arabic speaking countries are known to be diglossic communities (Ferguson, 1959). Modern Standard Arabic (SA henceforth) and at least one vernacular exist in all Arab communities (Schmitt, 2020). Tarabya et al. (2021) indicate that there is a language variety in individual response variance and accuracy between Literary Arabic (LA) and Standard Arabic (SA). Al-Rubaat and Qarqaz, (2019) indicated that there is a strong relationship between the temporary dialect of people from Skaka region (northern part of Saudi Arabia) and the SA in certain 
cases, however, the relationship is also found to be weak in other cases. The interaction between the two varieties is high and speakers tend to vary in their speech leading to phonological variation (Bassiouney, 2020). For example, the voiceless interdental fricative in $/ \theta / \mathrm{SA}$ is realized as $/ \mathrm{t} /$ in Jordanian Arabic (Glanville, 2018). However, speakers tend to use both standard and dialectal forms in their speech (Hammoudi \& Dendane, 2021). Many studies have looked at phonological variation in such communities, but they are limited to certain regions such as Jordan (Al-Wer, 1991), Syria (Daher, 1997), Iraq (Bakir, 1986) and many more. The majority of these studies focus on one or two non-linguistic factors such as age and/or gender. There have been a limited number of research studies which have focused on phonological variation in the Kingdom of Saudi Arabia (KSA). In addition, the comparison between two regions has rarely been made. Thus, this paper aims to focus on the phonological variation in the spoken Arabic of two regions: Jeddah, Saudi Arabia and Cairo, Egypt. The Arabic spoken in Jeddah is called the Hijazi Arabic (HA henceforth) and in Cairo it is called Cairene Arabic (CA henceforth). One phonological variable will be tested: the voiceless uvular stop /q/. The /q/ is used in SA. However, in dialectal Arabic, the variant in HA is the voiced velar stop /g/. In CA, the variant of /q/ is a glottal stop $/$ / /. Speakers tend to use the standard and/or dialectal forms in their speech. Some studies argue that switching between the two forms is to accommodate their listeners (Abu-Melhim, 1991). However, that is not always the case. As such, this paper also examines the role of age, sex, educational level and region on this variation. In what follows from the latter, are six sections organized as follows: Section 2 explores the literature relevant to phonological variation in Arabic. The hypotheses of the study will be detailed in Section 3. Section 4 details the method used to examine the variation in question. Sections 5 and 6 show the results of the study and discuss the results and Section 7 draws upon the conclusions of the study.

\section{Literature Review}

The previous studies that looked at phonological variation in Arabic either focus on a specific region or test one or two non-linguistic variables. The first study was conducted by Bakir (1986). Bakir (1986) examines whether men and women use standard Arabic forms in their speech or not. He tested twenty college students (10 male and 10 female) from the city of Basra, Iraq. The data he collected were based on two-hour recorded sessions amongst the participants. Bakir (1986) looked at three different variables. Each variable had two variants (standard and dialectal). He examined the use of $/ \mathrm{k} / \mathrm{vs}$. $/ \mathrm{t} /$ / passivation in Standard Arabic and Iraqi Arabic and the complementizer -an (whether overt or not) in both forms. The results of his study indicated that men use the standard $/ \mathrm{k} / \mathrm{va}-$ riable $73 \%$ of the time while women use it only $51 \%$. In passivation, men use the standard passive variable (57\%) more than women (23\%). Finally, men use -an $39 \%$ of the time while women only use it $26 \%$ of the time. The results of Bakir's 
(1986) study indicated that men use the standard variant more than women. Bakir (1986) attributes his findings to the status of women in the Arab world. Women are not required to communicate with the outside world compared to men. Even if a woman was educated and worked, there are limitations on the type of careers she can have. Because women are not participating in contexts that require a certain prestige, they will not use a linguistic form that is used in those contexts. Therefore, women use the dialectal form more than the standard (Ismail, 2012).

A different study that was conducted by Daher (1997) studies the phonological variation in Syrian Arabic. He also examined the effect of education level (elementary school, high school or college), gender and age (three different age groups) on this variation. The linguistic variables that he studied were the interdental fricatives $/ \theta /$ and $/ ð /$, the uvular stop /q/ and two diphthongs /aw/ and /ay/. The interdental fricatives are the standard forms and are realized as /s/ and /z/ respectively. The uvular stop /q/ is realized as / $/$ in Syrian Arabic and the diphthong /aw/ is [oo]. Finally, the diphthong [ay] is realized as [ee] in Syrian Arabic. Daher (1997) collected his data from twenty-three males men that represent the three different educational levels and age groups. The tokens were collected from thirty hours of face-to-face interviews with the participants.

Daher (1997) results, with respect to the interdental fricatives, indicate that women use the dialectal variant $/ \mathrm{s} /$ and $/ \mathrm{z} /$ more than the standard form. They used $/ \theta /$ and $/ ð / 4.5 \%$ and $6.6 \%$ of the time. When looking at the three different age groups and variable choice, the youngest group (age 15 - 24) has the lowest percentage of using the standard forms $(5.9 \%$ for $/ \theta /$ and $7.7 \%$ for $/ ð /$ ). Finally, the participants with the highest level of education have the highest percentage in using the standard variants. Daher (1997) also examined the use of /q/ vs. / $/$. The results show that males have a higher frequency in the use of the standard form /q/ (52.9\% vs. $42 \%)$ than females. With respect to age, the middle group (ages 25 - 39) use /q/ more frequently than the other two groups. The group with the highest level of education uses the standard variant/q/ more frequently (61\%). Finally, Daher (1997) looks at the distribution of /aw/ and /ay/ with respect to age, gender and educational level. The results show that men, the middle age group and highly educated people use the standard forms /aw/ and /ay/ more frequently than the dialectal variants. Based on his findings, Daher (1997) concludes that women use the dialectal variant more often than men. He also highlighted that there is a high correlation between the use of the standard variants and educational level. This can be seen in both men and women. Finally, the middle age group (ages 25 - 39 years old) is more likely to use the standard variants than the younger (15 - 24 years old) and older age groups (41 - 70 years old).

A study that focused on social class and gender differences was done by Haeri (1997). She examined the variables of apical palatalization, pharyngealization and the use of the variable /q/ in Cairene Arabic. An example of apical palatali- 
zation in Cairene Arabic is changing a / $\mathrm{t} /$ to a $/ \mathrm{t} \mathrm{f} / \mathrm{such}$ as the word bin[t] "girl" to bin[t $\mathrm{t}]$. Also, the use of pharyngeals in Arabic is considered to be the standard variant. However, in Cairene Arabic, many produce them as regular stops / $t /$ or /d/ or they are not as strong as the standard form. As for the variable /q/, it is realized as a glottal stop in Cairene Arabic. As such, Haeri (1997) examined these variables in relation to gender and social class. She found that men use strong pharyngealization and women have weak pharyngealization. In addition, the upper middle class has weak pharyngealization regardless of gender. The results for palatalization indicate that lower middle-class women are the highest users of the palatalized variant. Finally, with regards to the /q/ variable, Haeri (1997) found that in general men use the standard /q/ variant more than the glottal stop. However, the results also show that men from a higher social class use the standard form more than the glottal stop. Women do not use the /q/ as frequent as men, but there is a correlation between social class and the use of the standard form. Her findings indicate that women use the standard variants less frequently than men do because women do have access to education and professional life like men do. Finally, Haeri (1997) states that: "women who have equal levels of education to men use features of classical Arabic significantly less than men do" (p. 307).

Based on the previous studies, one can conclude that there is a correlation between gender, educational level and social class and the selection of which variant to use. Men use the standard variant more than women. Also, the higher the educational level, the more the standard variant will be used. Finally, the higher the social class, the more speakers will use the standard forms.

\section{Hypotheses}

The hypotheses being tested on /q/ variation in Arabic are:

1) Men are more likely to use the standard variant /q/ than women.

2) There is a positive correlation between the use of the standard variant and the speaker's level of education.

3) Younger speakers will use the non-standard variant more than older speakers.

4) Speakers from Jeddah will use the /g/ variant and speakers from Cairo will use the / $/$ / variable.

\section{Methodology}

\subsection{Participants}

The participants who agreed to take part in this study were from two regions: Jeddah and Cairo. They were from different age groups and educational backgrounds. Table 1 is a summary of the participants' demographic information.

\subsection{Data Source}

The primary gathered data was collected via semi-structured interviews with the 
Table 1. Participants' demographic information.

\begin{tabular}{ccccc}
\hline Speaker & Age & Gender & Region & Educational Level \\
\hline 1 & 57 & Male & Jeddah & Some college \\
2 & 55 & Female & Jeddah & Some high school \\
3 & 23 & Female & Jeddah & College \\
4 & 21 & Female & Jeddah & College \\
5 & 18 & Female & Jeddah & Some college \\
6 & 47 & Female & Cairo & High School \\
7 & 21 & Male & Cairo & Some College \\
8 & 18 & Female & Cairo & Some College \\
\hline
\end{tabular}

participants who agreed to take part in this study. The interviews were audio recorded on the researcher's own digital recorder and later transcribed verbatim and the text analysed. The researcher did not interfere nor hint any researchspecific aims so as not to influence the speech by each participant and phonological characteristics produced by the participants.

\subsection{Procedure}

The interviews conducted were based on Labov (1966) sociolinguistic interviews method. The tasks used in this study to collect the data were a word list and a reading paragraph. The word list consisted of forty-three words. Twenty-three of the words elicited the variable /q/. Eight words elicited the variable /q/ word initially, eight words medially, and seven words finally. The remaining words were used as fillers that did not have /q/. The second task was a reading passage that contained words with the variable /q/. The reading passage was a story of a boy who loved music. The passage contained nineteen words that elicited the variable /q/. One final task that was supposed to be carried out was casual speech. The researcher intended to ask a few questions after the two tasks mentioned above. However, the first four participants declined to engage in the third task and therefore, only the two tasks mentioned above were conducted. All the interviews were recorded using a digital recorder. The researcher extracted the variables in question from the recordings and coded them on an excel spreadsheet for analysis.

\section{Results}

The results are divided into two sections: The HA results and the CA results. Under each section, the results of the study are presented by the variant in question, /q/, and the three non-linguistic factors: age, gender and education level. The results are analysed based on the percentage use of the SA variable /q/ and the dialectal variable / $/ \mathrm{/}$ or $/ \mathrm{g} /$. In addition, the results demonstrate the correlation between the choice of variable and age, gender and educational level. 


\subsection{CA Results}

The results of CA are divided into three sub-sections based on the non-linguistic factors in question: age, gender and educational level.

\subsection{Age}

The results indicate that younger speakers use the standard variable less than older speakers. Younger speakers use /q/ 67\% of the time while older speakers use it $79 \%$. Table 2 summarizes the mean percentages of the use of /q/ vs. / $/$ and its relation to age.

\subsubsection{Education Level}

The speakers from the CA dialect are divided into two levels of education: speakers with a high school degree and are currently enrolled in college and speakers who only finished high school. The results indicate that speakers who are not enrolled in college or did not complete a higher degree use the standard variant $79 \%$ of the time. Table 3 below summarizes mean percentages of the use of /q/ vs. / $/$ / and its relation to educational level.

\subsubsection{Gender}

The data indicates that male speakers use the standard variant /q/ more than females. Male speakers use it $74 \%$ of the time while females use it only $69 \%$. However, no statistical analysis tests have been conducted to determine in the difference is significant or not. Table 4 is a summary of the mean percentages of the use of /q/ vs. / $/$ in relation to sex.

\subsection{HA Results}

The results in HA indicate that there is not much variation. If we look at age, we

Table 2. Mean percentage of /q/ and / / by age in CA (word list and reading tasks).

\begin{tabular}{cccc}
\hline & Tokens & /q/ & $/ \mathrm{R} /$ \\
\hline$<25$ & 86 & 67 & 33 \\
$>40$ & 43 & 79 & 21 \\
\hline
\end{tabular}

Table 3. Mean percentage of /q/ and / $/$ by education level in CA (word list and reading tasks).

\begin{tabular}{cccc}
\hline & Tokens & /q/ & $/$ / \\
\hline Some college & 86 & 67 & 33 \\
High School & 43 & 79 & 21 \\
\hline
\end{tabular}

Table 4. Mean percentage of /q/ and / $/$ by sex in CA (word list and reading tasks).

\begin{tabular}{cccc}
\hline & Tokens & /q/ & $/$ / / \\
\hline Male & 43 & 74 & 26 \\
Female & 86 & 69 & 31 \\
\hline
\end{tabular}


find that there is a small difference between older and younger speakers. Younger speakers use the standard variant /q/ $100 \%$ of the time while older speakers vary a little in their speech and use /q/ $94 \%$ of the time. In addition, the educational level does not seem to influence the speakers' choice of variable. In HA, there were three different levels of education (some high school, some college, and college graduates). The only group that had some variation was the group (some college). The speakers in that category used the standard variant /q/ 94\% of the time. Table 5 and Table 6 are a summary of the results by age and educational level.

When looking at gender, we can see a difference between male and female speakers. Male speakers use the standard /q/ less than females. Males use /q/ 88\% of the time while females use it $100 \%$. Table 7 summarizes the mean percentages by gender.

\subsection{Results' Summary}

As per the statistical analysis above, it can be seen that age and educational level in both CA and HA generated little variation in the production of standard /q/ compared to the variant /q/, while the gender element played a part in the production of standard /q/ compared to the variant /q/. Male speakers use the standard /q/ less than females.

\section{Discussion}

The analysis of the results shows that there is more variation in CA speakers than in HA speakers. Referring back to first hypothesis of the study: Men are

Table 5. Mean percentage of /q/ and /g/ by age in HA (word list and reading tasks).

\begin{tabular}{cccc}
\hline & Tokens & /q/ & /g/ \\
\hline$<25$ & 129 & 100 & 0 \\
$>40$ & 43 & 94 & 6 \\
\hline
\end{tabular}

Table 6. Mean percentage of /q/ and /g/ by education level in HA (word list and reading tasks).

\begin{tabular}{cccc}
\hline & Tokens & $/ \mathrm{q} /$ & $/ \mathrm{g} /$ \\
\hline Some high school & 43 & 100 & 0 \\
Some college & 86 & 94 & 6 \\
College & 86 & 100 & 0 \\
\hline
\end{tabular}

Table 7. Mean percentage of /q/ and /g/ by sex in HA (word list and reading tasks).

\begin{tabular}{cccc}
\hline & Tokens & /q/ & $/ \mathrm{g} /$ \\
\hline Female & 172 & 100 & 0 \\
Male & 43 & 88 & 12 \\
\hline
\end{tabular}


more likely to use the standard variant /q/ than women, this can be seen evident in CA. Male speakers from Cairo tend to use the standard form /q/ more than the non-standard form. As such, the results of this study confirm the first hypothesis. In addition, the results also give additional support to other studies that looked at gender differences such as Bakir (1986), Abdel-Jawad (1983) and Haeri (1997). All these studies found that men use the standard forms more than women. However, the results from the HA do not support the first hypothesis. We find that female speakers use the standard variant /q/ more than male speakers in HA. The results from HA support Labov (1966) where women are more conservative than men and use the standard linguistic forms more than men. Gender differences occur in Arabic however it depends on the region. Male speakers from Cairo will use the standard forms while male speakers from Jeddah will use the non-standard form.

Looking at the second hypothesis of the study: There is a positive correlation between the use of the standard variant and the speaker's level of education, the results of the study do not confirm this hypothesis. In both CA and HA results, the differences between the levels of education are not high. In addition, the comparison between the different levels of education was based on mean percentages. No statistical analysis was conducted to determine if the difference was significant or not. In general, educational level does not seem to influence the choice of variant the speaker chooses. The results of this study do not give additional support to previous studies that found that there is a positive correlation between the speaker's level of education and use of the standard variant such as that of Daher (1997).

The third hypothesis of the study was: younger speakers will use the nonstandard variant more than older speakers. The results analysed from the gathered data from the CA Arabic speakers support this hypothesis. The younger group used /q/ $67 \%$ of the time while the older group used it $79 \%$ of the time. On the other hand, the results from HA do not support this hypothesis. The younger speakers used the standard variant $100 \%$ of the time while the older speakers used it $94 \%$. The issue here again is whether this difference is statistically significant or not.

The fourth hypothesis was: Speakers from Jeddah will use the /g/variant and speakers from Cairo will use the / $/$ / variable. This hypothesis is supported in this study. In CA, when the speaker used the non-standard form, they would use the glottal stop $100 \%$ of the time. In HA, when the speaker used the non-standard form, they would use the voiced velar stop $/ \mathrm{g} /$.

Finally, one interesting observation to note is that in HA, the highest variation occurs between male and female. Males only use the standard variant $88 \%$ of the time. However, this variation occurred with words that had a higher frequency in the language. For example, one word from the reading passage was [qarura] "bottle". This word is very frequent therefore the speaker used the non-standard variant for $/ \mathrm{q} /$ which is [g]. When faced with a less frequent word like [qanun] "law", the speaker used [q] i.e. the standard variant. This observation leads us to 
think that frequency of lexical items may a play a role in the choice of variant the speaker uses.

To get a better understanding of the nature of variation in CA and HA, one must consider methodological implications. The participants in the study did not agree to a spontaneous production task. Spontaneous productions will help us get a better understanding on the nature of variation in their speech because they are less attentive to their speech. A future direction for this study is to gather data from causal speech. In addition, a look at word frequency in Arabic is another direction for future studies.

\section{Conclusion}

The results of this study did not support all the hypotheses presented earlier. However, the results indicate that variation in CA is higher than HA. In addition, male speakers in CA use the standard variant more than females, but opposite findings were found for HA. Also, younger speakers in CA tend to use the non-standard variant more than older speakers. More data needs to be gathered from different tasks to truly understand the nature of variation in these two varieties. Also, the results of the study also support findings from previous findings on Arabic and other languages as well. Overall, this study serves as a starting point in looking at phonological variation in Arabic specifically in Hijazi Arabic where not much has been done.

Thus, this study confirms the hypothesis of the production of phonological variances in the production of standard /q/ due to the gender element, however, it does not offer support to the hypothesis that indicates positive correlation between the use of the standard variant and the speaker's level of education. The study does not also offer support to the hypothesis which indicates that the age elements play a role in the production non-standard variant produced by the younger generation compared to the older generation. Finally, the study offers support to the hypothesis which indicates that speakers from Jeddah (HA) will use the /g/ variant and speakers from Cairo (CA) will use the /?/ variable.

\section{Conflicts of Interest}

The author declares no conflicts of interest regarding the publication of this paper.

\section{References}

Abdel-Jawad, H. (1983). Sex Differentiation and Linguistic Variations. A Case Study: Spoken Arabic in Amman. ERIC.

Abu-Melhim, A. R. (1991). Code-Switching and Linguistic Accommodation in Arabic. In B. Comrie, \& M. Eid (Eds.), Perspectives on Arabic Linguistics III: Papers from the Third Annual Symposium on Arabic Linguistics (pp. 231-250). John Benjamins Publishing Company. https://doi.org/10.1075/cilt.80.15abu

Al-Rubaat, A., \& Qarqaz, A. A. (2019). Supra Segmental Phonology in Skaka Dialect and Its Relation to the Modern Standard Arabic. Open Journal of Modern Linguistics, 9, 
330-342. https://doi.org/10.4236/ojml.2019.95027

Al-Wer, E. E. (1991). Phonological Variation in the Speech of Women from Three Urban Areas in Jordan. Ph.D. Thesis, University of Essex.

Bakir, M. (1986). Sex Differences in the Approximation to Standard Arabic: A Case Study. Anthropological Linguistics, 28, 3-9.

Bassiouney, R. (2020). Arabic Sociolinguistics: Topics in Diglossia, Gender, Identity, and Politics. Georgetown University Press. https://doi.org/10.2307/j.ctv10kmbxg

Daher, J. (1997). Phonological Variation in Syrian Arabic: Correlation with Gender, Age, and Education. Amsterdam Studies in the Theory and History of Linguistic Science Series, 4, 239-272.

Ferguson, C. A. (1959). Diglossia. Word, 15, 325-340. https://doi.org/10.1080/00437956.1959.11659702

Glanville, P. J. (2018). The Lexical Semantics of the Arabic Verb. Oxford University Press. https://books.google.co.uk/books?id=FE1YDwAAQBAI https://doi.org/10.1093/oso/9780198792734.001.0001

Haeri, N. (1997). Sociolinguistic Market of Cairo. Routledge.

Hammoudi, K., \& Dendane, Z. (2021). Directions of Dialect Change in the Mashreq and the Maghreb: Case of Arabic Varieties Spoken in Amman and Tlemcen Speech Communities.

Ismail, M. A. (2012). Sociocultural Identity and Arab Women's and Men's Code-Choice in the Context of Patriarchy. Anthropological Linguistics, 54, 261-279.

https://doi.org/10.1353/anl.2012.0018

Labov, W. (1966). The Social Stratification of English in New York City. Center for Applied Linguistics.

Schmitt, G. A. (2020). Relevance of Arabic Dialects: A Brief Discussion. In S. Brunn, \& R. Kehrein (Eds.), Handbook of the Changing World Language Map (pp. 1383-1398). Springer. https://doi.org/10.1007/978-3-030-02438-3 79

Tarabya, B. M., Khateb, A., \& Andria, S. (2021). Processing Printed Words in Literary Arabic and Spoken Arabic: An fNIRS Study. Open Journal of Modern Linguistics, 11, 461-483. https://doi.org/10.4236/ojml.2021.113035 